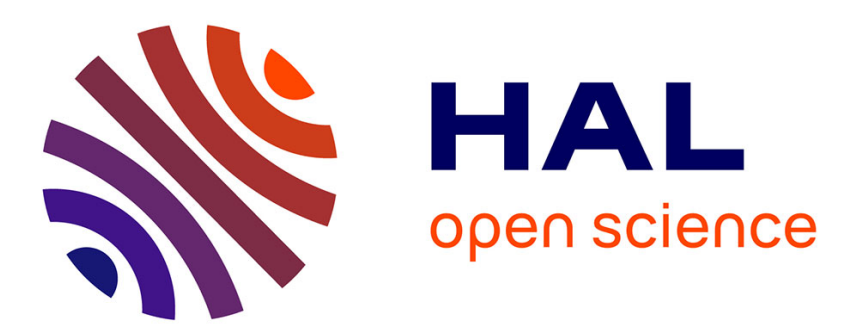

\title{
Learning the Spherical Harmonic Features for 3-D Face Recognition
}

Peijiang Liu, Yunhong Wang, Di Huang, Zhaoxiang Zhang, Liming Chen

\section{To cite this version:}

Peijiang Liu, Yunhong Wang, Di Huang, Zhaoxiang Zhang, Liming Chen. Learning the Spherical Harmonic Features for 3-D Face Recognition. IEEE Transactions on Image Processing, 2013, 22, pp.914-925. 10.1109/TIP.2012.2222897 . hal-01351702

\section{HAL Id: hal-01351702 https://hal.science/hal-01351702}

Submitted on 13 Mar 2017

HAL is a multi-disciplinary open access archive for the deposit and dissemination of scientific research documents, whether they are published or not. The documents may come from teaching and research institutions in France or abroad, or from public or private research centers.
L'archive ouverte pluridisciplinaire HAL, est destinée au dépôt et à la diffusion de documents scientifiques de niveau recherche, publiés ou non, émanant des établissements d'enseignement et de recherche français ou étrangers, des laboratoires publics ou privés. 


\title{
Learning the Spherical Harmonic Features for 3D Face Recognition
}

\author{
Peijiang Liu, Yunhong Wang*, Member, IEEE, Di Huang, Member, IEEE, \\ Zhaoxiang Zhang, Member, IEEE, and Liming Chen, Member, IEEE,
}

\begin{abstract}
In this paper, a competitive method for 3D face recognition (FR) using Spherical Harmonic Features (SHF) is proposed. With this solution 3D face models are characterized by the energies contained in spherical harmonics with different frequencies, thereby enabling the capture of both gross shape and fine surface details of a 3D facial surface. This is in clear contrast to most 3D FR techniques which are either holistic or feature-based using local features extracted from distinctive points. First, 3D face models are represented in a canonical representation, namely Spherical Depth Map (SDM), by which SHF can be calculated. Then, considering the predictive contribution of each SHF feature, especially in the presence of facial expression and occlusion, feature selection methods are used to improve the predictive performance and provide faster and more cost-effective predictors. Experiments have been carried out on three public 3D face datasets, namely SHREC2007, FRGC v2.0 and Bosphorus, having increasing difficulties in terms of facial expression, pose and occlusion, and which demonstrate the effectiveness of the proposed method.
\end{abstract}

\section{Index Terms}

3D face recognition, Spherical Depth Map, spherical harmonics, feature selection

\footnotetext{
* Corresponding author.

P. Liu, Y. Wang, D. Huang and Z. Zhang are with Laboratory of Intelligent Recognition and Image Processing, Beijing Key Laboratory of Digital Media, School of Computer Science and Engineering, Beihang University, Beijing, China; and also with the State Key Laboratory of Virtual Reality Technology and Systems, Beihang University, Beijing, China (e-mail: pjliu@cse.buaa.edu.cn; yhwang@buaa.edu.cn; dhuang@buaa.edu.cn; zxzhang@buaa.edu.cn.)

L. Chen is with University of Lyon, CNRS, Ecole Centrale de Lyon, LIRIS UMR 5205, F-69134, France (e-mail: liming.chen@ec-lyon.fr)
} 


\section{INTRODUCTION}

The face is a natural and non-intrusive biometrics which can be easily acquired contactlessly. Unfortunately, despite the great strides made over the last thirty years, face recognition (FR) in $2 \mathrm{D}$ is still a great challenge due to factors as diverse as illumination variations, pose changes and facial expressions [1]. With the advent of readily available 3D scanners, there has been an increasing interest in using $3 \mathrm{D}$ data instead of, or in addition to $2 \mathrm{D}$ images to overcome the limitations in 2D FR, in particular viewpoint and lighting variations[2], and to achieve better recognition accuracy than 2D FR [3], [4], [5]. As 3D FR operates on 3D face scans delivered by 3D sensors and they are supposed to convey the accurate shape information of facial surfaces, 3D FR needs to address two kinds of challenges in order to meet real-world application requirements, namely $3 \mathrm{D}$ data sensing in unconstrained settings and improved algorithms with high recognition accuracy while displaying strong robustness to various variations and factors of disturbance such as facial expressions, partial face data and pose changes [4]. In this paper, we concentrate on the second challenge and propose a competitive method for $3 \mathrm{D}$ face recognition using Spherical Harmonic Features.

\section{A. Related work}

Face recognition is a pattern recognition problem which requires a proper representation of face data highlighting interclass differences while decreasing intra-class variations in order to facilitate the later stage of matching and recognition. In this subsection, we overview major 3D facial representations and the corresponding matching algorithms. State of the art on feature selection can be found in [6], [7], [8], [9].

1) 3D Face Representations and registration: 3D face data are the scans delivered by 3D sensors which accurately capture the shape information of facial surfaces. As such, 3D FR can theoretically make use of any existing shape representation used in 3D shape retrieval, e.g., Extended Gaussian Image [10], Shape Index [11], Spin Image [12], Shape Histogram [13], Shape Distribution [14], Spherical Harmonic Representation [15], Representation based on surface reconstruction [16]. Nevertheless, 3D face data has also its own particularities which need to be taken into account. First of all, all 3D face scans are similar in their configuration. As compared to the general issue of 3D shape-based retrieval which performs retrieval of fundamentally different object classes, e.g., car, table, glass, etc., 3D FR performs the recognition within face models of a 
same class. As a result, a proper 3D face representation needs to highlight the distinctiveness of 3D facial surfaces for a better discriminating power. Moreover, some prior anatomical knowledge of human faces can be utilized to enhance the performance of representation. Besides, 3D face scans as delivered by current 3D sensors are generally partial face models having pose variations and facial expressions.

3D FR algorithms can directly operate on the raw 3D point clouds of 3D face scans or depth maps, also called range images, extracted from the latter in projecting the $3 \mathrm{D}$ depth data to a $2 \mathrm{D}$ image according to the $\mathrm{z}$-depth of the 3D points. However, a growing number of works in literature have investigated alternative 3D facial representations robust to facial expressions and/or highlighting the distinctiveness of human faces, including the isometric deformations of a facial surface [17], geometry image [18], indexed facial level curves [19], ridge image [20], Signed Shape Difference Map (SSDM) [21], Multi-Scale Extended LBP (MS-eLBP) [22], to name a few.

Most 3D FR techniques require that 3D face scans be aligned before the generation of a appropriate facial representation and the extraction of facial features for matching [3], [4]. Existing techniques on registration still need to be improved regarding their robustness and accuracy. Gokberk et al. [23] reviewed popular algorithms of 3D face registration and recognition based solely on shape information. In the thesis of Jebara [24], symmetric blob detection, limb extraction and signature analysis were used to locate eyes, mouth and nose respectively. But its success largely depends on anthropometry knowledge. Gordon [25] used a cylindrical coordinate system to represent depth information under the assumption that the head was vertical. Colbry et al. [26] used shape index to detect the anchor points in 3D face scans and achieved $99 \%$ success rate in finding the anchor points in frontal images and $86 \%$ success rate in scans with large variations in pose and expression. Conde et al. [27] successfully applied Spin Image to facial normalization and face verification. Tang et al. [28] found the reference point and nose line for face registration by computing the symmetry plane of the nose region using prior anatomical knowledge. Pears et al. [2] gave an elaborate review of related work and applied radial basis function $(\mathrm{RBF})$ to creating a pose-normalized depth map.

2) Recognition algorithms: 3D FR algorithms can be categorized according to the nature of their matching strategy, either holistic [29], [19], [30], [31], [32], [20], [33], [21], [34] or feature-based [35], even though this is highly dependent on the facial features extracted from 
a given facial representation. In this section, we only discuss some new state-of-the art 3D FR techniques as a complement to the existing elaborate reviews [3], [4], [5].

Haar and Veltkamp [29] propose a 3D face matching framework by combining three curves of 45 face samples in SHREC2007 [36] database and achieve the highest mean average precision (MAP) of 0.78 .

Samir et al. [19] propose an intrinsic Riemannian framework for the analysis of facial surfaces. Firstly, they represent facial surface as indexed facial curves derived from a surface distance function. Then, a Riemannian metric denoted the differential geometry of face shapes is achieved for the following purposes: computing the distances between faces, finding optimal deformations between faces, defining and computing the average of a given set of faces.

Al-Osaimi et al. [30] present an automatic approach which can accurately discriminate between expression deformations and interpersonal disparities. An expression deformations pattern is first learnt from training data in PCA eigenvectors, by which they compute the similarity measures between 3D faces and hence recognize faces under any facial expression. The verification rates achieved on the FRGC2.0 database at $0.1 \%$ FAR are $98.35 \%$ and $97.73 \%$ for scans under neutral and non-neutral expressions respectively.

Boehnen et al. [31] propose a fast face recognition method based on a vector representation named 3D signature whose elements correspond to the fixed surface points in a face-centered coordinates system. The proposed method runs more than three orders of magnitude faster than a traditional ICP-based distance implementation, without sacrificing accuracy. Finally, they achieve an improvement of $0.2 \%$ to $0.5 \%$ more than the previous highest performing on FRGC2.0 by a new Match Sum Thresholding (MST) fusion approach.

$\mathrm{Xu}$ et $a l$. [32] propose a novel face recognition system combining local Gabor wavelets features extracted from depth and intensity images with a hierarchical feature selection scheme embedded in linear discriminant analysis(LDA) and AdaBoost learning when expression and pose variations are taken into account. Their experiments are performed on CASIA and FRGC2.0 databases. The highest recognition accuracy on CASIA is $98.3 \%$ on neutral face set and $79.0 \%$ on face set with large pose variations and smiling. The verification rate at $0.1 \%$ FAR in the protocol of neutral vs. all on FRGC2.0 is $97.5 \%$.

Mahoor et al. [20] propose a novel representation of 3D face called ridge image and utilize two tools: robust Hausdorff distance (HD) and iterative closest points (ICP), for matching them. 
Their experimental results are reported on the GavabDB and FRGC2.0 databases. For neutral frontal faces, the rank-one identification rate is $95 \%$ and $91.8 \%$ using ICP techniques on the two databases, respectively. For faces with expressions and pose variations in GavabDB, the identification rates are reduced to $83.6 \%$ and $88.6 \%$, respectively. The verification rates at $0.1 \%$ FAR for the ROC I, II, and III of the neutral versus neutral in FRGC2.0 are 90.69\%, 88.5\% and $85.75 \%$, respectively, which is better than the FRGC baseline.

Llonch et al. [33] represent 3D face as signals on the 2-sphere and then reduce the dimensionality with simultaneous sparse approximations and subspace projection which captures the salient facial characteristics for recognition. The experimental results conducted on FRGC1.0 show that their methods outperform classical state-of-the-art solutions that work with depth images.

Queirolo et al. [37] firstly register 3D range face images using a Simulated Annealing-based approach (SA). Then, the similarity measure of faces is obtained by combining the Surface Interpenetration Measures corresponding to four different face regions: circular and elliptical areas around the nose, forehead, and the entire face region. A verification rate of $96.5 \%$ at $0.1 \%$ FAR is achieved on FRGC2.0 database. The rank-one identification accuracy of $98.4 \%$ is reported on the same data set.

Wang et al. [21] propose collective methods for 3D face recognition. After a self-dependent alignment, 3D faces are represented as a mediate form named Signed Shape Difference Map (SSDM), based on which, three kinds of features are calculated and trained by respectively boosting for assembling three collective strong classifiers named Collective Shape Difference Classifiers. Their experiments conducted on FRGC2.0 with the standard protocol report three verification rates which are all better than $97.9 \%$ at $0.1 \%$ FAR, and a rank-one recognition rates of $98 \%$.

Berretti et al. [34] present a novel face representation in the form of graph, in which the nodes represent equal width iso-geodesic facial stripes and the arcs refer to a new descriptor of 3D Weighted Walkthroughs (3DWWs). The graph-based representation enables efficient face matching in large data sets with the support of appropriate index structures. Finally, the proposed method is evaluated on the FRGC2.0 and the SHREC08 databases and achieves competitive results.

Huang et al. [35] propose a biological vision-based facial description, namely Perceived Facial Images (PFIs), along with a local feature-based SIFT matching. They achieve $98 \%$ rank-one 
recognition rate on FRGC v2.0 and competitive results on the challenging 3D TEC dataset consisting of 3D scans of 107 pairs of twins [38].

Holistic matching schemes are based on holistic facial representations which make use of all the facial shape data for discrimination. They hence generally require an accurate normalization step with respect to pose and scale changes. The disadvantage is also that they are vulnerable to occlusions and facial shape deformations, e.g., facial expressions. Now feature-based approaches achieve the matching procedure through local features which are extracted typically from the prominent points on facial surfaces [39], [40], e.g., curvature extrema. Feature-based matching has the potential advantage of being robust to facial expression, pose and lighting changes and even to partial occlusions. The downside of this scheme is the difficulty in extracting sufficient informative feature points from similar or smooth 3D facial surfaces.

\section{B. Overview of the proposed approach}

In this paper, we propose Shape-based Spherical Harmonic Features (SHF) for 3D FR. These SHFs capture both gross shape and fine surface details of facial surfaces through the energies contained in spherical harmonics at different frequencies. They are computed on a canonical facial representation, namely Spherical Depth Map (SDM). Because the predictive contribution of each feature in SHF is different, especially in the presence of facial expressions and possible partial occlusions, we further investigate feature selection methods to improve the predictive performance and provide faster and cost-effective predictors.

Shape-based Spherical Harmonic Features were successfully applied to 3D shape-based interclass retrieval [41], [15]. When applying SHF to 3D FR which is an intra-class problem, it is necessary to properly address the following two issues. The first is how to reliably calculate SHF for a 3D face scan. Funkhouser et al. [41] achieves a translation and pose normalized 3D shape descriptor by moving the model so that the center of mass lies at the origin and by discarding phase information. However, such an approach is only suited to the context of wholeobject to whole-object matching [15]. In the context of 3D FR which has to perform partial 3D face matching, the center of mass translation can not be expected to provide optimal results. Therefore, we propose a novel representation of 3D face scans, subsequently named Spherical Depth Map (SDM), to provide a canonical representation for arbitrary 3D scans represented in unstructured point clouds. The second issue is how to improve the discirminating ability of 
SHF for 3D FR. Some researchers calculate the L2-norm of each frequency component at each radius in order to obtain a rotation invariant shape descriptor, however, the information lost will seriously deteriorate the performance of face recognition [41], [15]. Therefore, original SHF is used in our method after an exact face alignment based on Spherical Depth Map [42].

Feature selection aims to find faster and more effective predictor while improving or maintaining the prediction performance [6]. In contrast with other subspace-based dimensionality reduction techniques, e.g., Principal Component Analysis (PCA) or Independent Component Analysis (ICA), feature selection techniques do not change the original representation of the features, but merely select a subset of them. As a result, feature selection techniques preserve the original semantics of the features, which offers the advantage of interpretability [43]. According to the relations with predictor, feature selection algorithms broadly fall into two categories: filter and embedded methods. In this study, the representative methods of two categories are investigated in order to select the most relevant SHF to a given predictor.

The proposed method consists of two stages: an off-line training stage for the selection of relevant SHF features and an online 3D FR stage as Fig.1 illustrates. In both off-line and online stage, 3D face data are first preprocessed, in particular to remove spikes and fill holes. Then face-aligned Spherical Depth Map is generated by the proposed pipeline in our early work [44]. In the training stage (off-line), we calculate a large set of SHF on training face data for the purpose of relevant feature selection. In the online 3D FR stage, only the selected subset of SHF is involved in classification, thereby significantly reducing the computational burden so that the process can work in real time.

The contributions of this work can be summarized as follows: (1) we have proposed the use of Spherical Harmonic Features (SHF) computed on a canonical facial representation, namely Spherical Depth Map (SDM), to capture both gross shape and fine surface details of 3D face models. (2) A novel framework for 3D FR using SHF and feature selection methods have been defined, yielding competitive performance on three public 3D face datasets. Under the combined effect of the discriminating power of SHF and feature selection methods, the proposed framework proves effective and displays robustness to facial expression and occlusion as shown by the experimental results on FRGC v2.0 and Bosphorus. Furthermore, the feature selection methods have helped to decrease the computational complexity by a factor of 3 .

The rest of the paper is organized as follows. Section II introduces the generation of SDM 


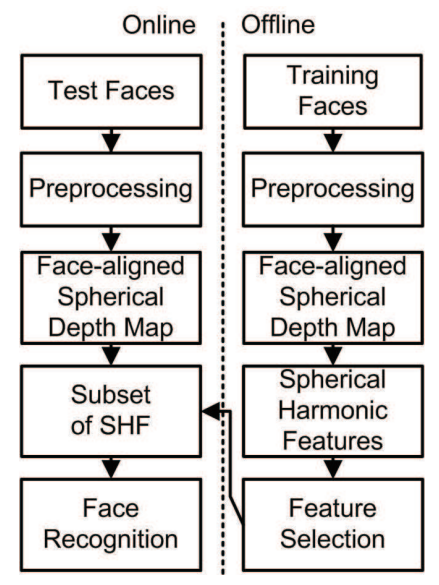

Fig. 1. The flowchart of our methods.

and its distinctive advantages compared with traditional range image. In Section III, we present Spherical Harmonic Features which are reliably calculated based on SDM. Section IV describes briefly three representative algorithms of feature selection method. The experimental results are then discussed in Section V. Finally, we conclude the paper in Section VI.

\section{SPHERICAL DEPTH MAP}

Before the calculation of Spherical Harmonic Features, 3D faces must be formalized as a spherical function with the same translation scale. The center of mass translation as proposed in [41], [15] only worked well in the context of whole-object to whole-object matching. In the context of 3D FR where we have only frontal facial surfaces, the center of mass translation cannot be the optimal solution. To overcome this defect, a novel method for face shape representation, namely Spherical Depth Map (SDM), is proposed in this paper, which translates the face shape so that the center of the fitting sphere rather than the center of mass is at the origin.

The basic assumption in the generation of a SDM associated with a 3D face scan is that the shape of human head can be viewed as a sphere. Although the human head has a shape rather close to an ellipsoid, which is also homeomorphic Riemannian manifold as the sphere, a spherical fitting has similar performance but less computational complexity.

The generation of a SDM is achieved through the following steps as Fig.2 shows. More details can be found in our earlier work [42], [44].

(1) A sphere is fitted to the point clouds of a 3D face scan as shown in Fig.3. 


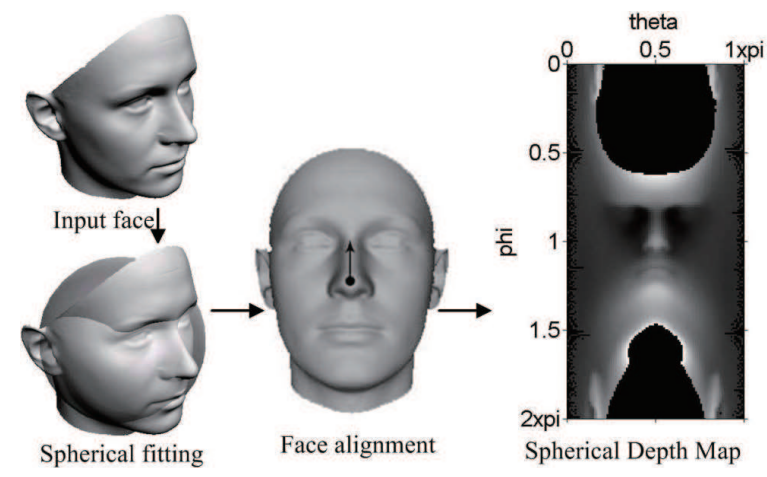

Fig. 2. The generation of face-aligned SDM.

(2) 3D face shape is translated so that the center of the fitted sphere is at the origin.

(3) The scale of Cartesian coordinates is normalized into an uniform interval.

(4) The Cartesian coordinates of the input face point clouds is transformed into spherical coordinates $(r, \theta, \varphi)$.

(5) To calculate the SHF, the face pose is automatically normalized into a uniform orientation [44].

By interpolation on a grid of $\theta$ and $\varphi$, the SDM of a 3D face scan can be viewed in a rectangle of $[\theta(0 \sim \pi), \varphi(0 \sim 2 \pi)]$ as shown the last image in Fig.2.

Fig. 3 shows the three-view drawings of a head shape after spherical fitting. As we can see, the face shape can be now described using a coordinate system attached to the face shape.

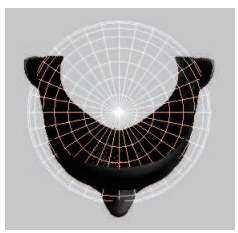

(a) top view

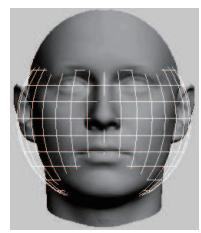

(b) front view

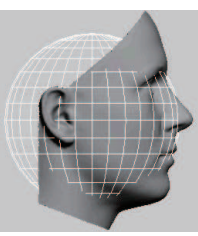

(c) side view

Fig. 3. Three-view drawings of face shape with fitting sphere.

The Iterative Closest Point (ICP) algorithm has proved its effectiveness for 3D face alignment by iteratively minimizing the Mean Square Error (MSE) of all point pairs between two face scans [45]. However, the original ICP algorithm cannot handle the case of 3D face scans with different scales. Fig.4 shows an example of face scale variations in which all faces are from the 
same subject in the FRGC2.0 database. Therefore, we achieve the generation of face-aligned SDM of 3D face using the method that we proposed in an earlier work [44].

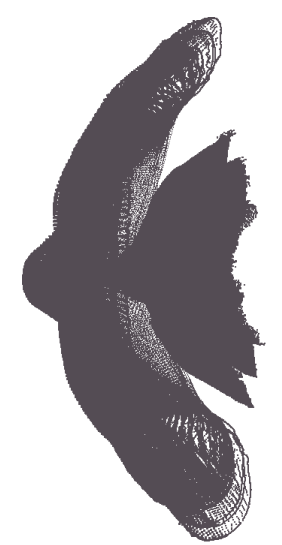

Fig. 4. Face scale variations in FRGC2.0 database. Faces are showed in same coordinates system.

SDM can work quite well on uncontaminated 3D face scans like the general model as shown in Fig.3. Nevertheless, If an input face scan encompasses foreign matters such as shoulder part or serious occlusion, the corresponding SDMs will be impacted as shown in Fig.5.

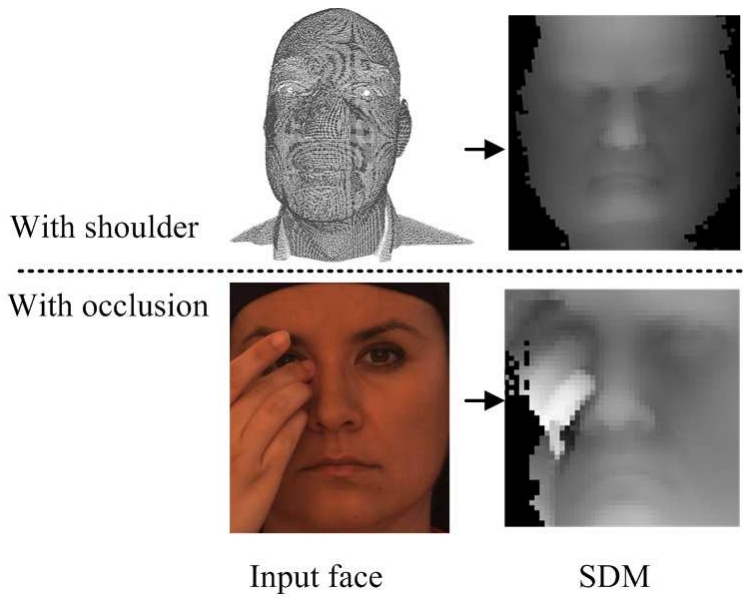

Fig. 5. The influence of the foreign matters on SDM. As the second column shows, the spherical scale of 3D face in SDM is variant because of the foreign matters.

To improve the accuracy of face alignment for the generation of robust SDMs, we made use of another landmark on 3D face scans in addition to the nose-tip. Although many other landmarks 
can be used which can automatically be accurately located [46] [47], the lowest point between the nose-tip and the center of forehead is employed to control the scale of 3D face scans in this work. Based on the previous work, the accuracy of the face alignment is improved by the following process:

(1) Find the symmetry plane of the input 3D face scan.

(2) The point having the lowest spherical depth on the intersection of the symmetry plane and the input 3D face scan is located as the required additional landmark as Fig.6 shows.

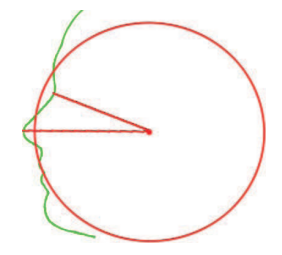

Fig. 6. The control of the scale of 3D face in SDM.

(3) The scale of the input 3D face scan is normalized by scaling the face so that the angle between the nose-tip and the detected landmark is equal to that of a reference model.

Although the asymmetry of human faces can be seen as a biometric trait for person identification [48], the preprocessing step of finding 3D face symmetry plane in this work is quite different from that work. We aim to locate a plane with which the input face can be divided into two relative symmetric parts for alignment. In fact, such information is also required in [48] in terms of "midline" to calculate the face asymmetry.

All of the above precessing is conducted automatically except a few failures caused by 3D face scans with an occlusion in the Bosphorus database. Finally, we obtain the face-aligned SDM of each 3D face model. Fig.7 shows several examples of face-aligned SDMs from the three databases.

The advantages of using SDMs can be summarized as follows:

- The generation of SDMs is a Mesh free method. The process of generating SDMs can simply operate on scattered point clouds of a $3 \mathrm{D}$ face scan, so no mesh or triangulation is required.

- Spherical scale invariance. Constrained by the configuration of the human head, the face area on the fitted sphere is invariant (about $\pi / 2 \times \pi / 2$ ). Therefore, the face scale is naturally 


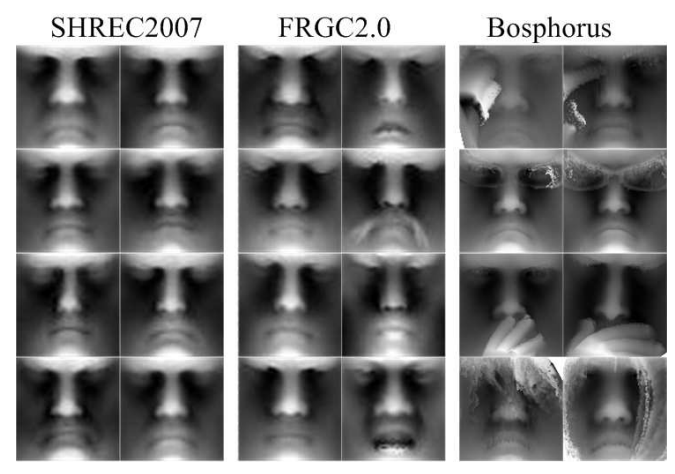

Fig. 7. SDM examples of 3D face models from SHREC2007 (left), FRGC v2.0 (middle) and Bosphorus (right). Face scans in FRGC2.0 have varied facial expressions whereas those in Bosphorus have varied occulisions. The faces in SDM are cropped by a square having the size of $\pi / 2 \times \pi / 2$.

normalized on the sphere.

- Dealing with face pose variation is as simple as a rigid rotation around the center of the fitted sphere.

- Object-centered shape representation. The face shape is described by a coordinate system attached to the $3 \mathrm{D}$ face. The origin of the coordinate system is the center of the fitted sphere which is derived from the face model itself.

- Complete shape representation. Only coordinate transformation (one-to-one mapping) is conducted on the point clouds during the generation process. There is no loss of information. As a result, the original face model can be completely reconstructed.

- Robustness to noise, small occlusion and expression variations. The center of the fitted sphere is calculated based on the global configuration of the head shape, which is insensitive to local variations caused by point-level noise, small occlusion or facial expression.

The original SDM associated with a 3D face scan is still a discrete representation, i.e. the points on a SDM bear no regular structure. This makes it difficult to be used directly. Nevertheless, by interpolation, an implicit function radius $=f(\theta, \phi)$ can be reconstructed from a given discrete SDM. Similar to range images, SDMs can be used as preprocessing step in many methods and applications, including Principal Components Analysis (PCA), Linear Discriminant Analysis (LDA), Gabor filter, Support Vector Machines (SVM), boosting methods, SIFT and so on. 


\section{SPHERICAL HARMONIC FEATURES}

Based on the SDM representation, with the same spherical coordinate system, it is natural to make use of spherical harmonics for the description of 3D facial surfaces. Although this technique has been introduced to characterize general 3D objects, using spherical harmonics for 3D facial surface description has not been investigated yet in the literature. Indeed, 3D FR is quite a different problem from that of general 3D object retrieval which is an inter-class retrieval problem in the sense that it needs to retrieve fundamentally different object classes, e.g., glass, chair, car. In the case of 3D FR, one needs to recognize different instances of the same class, i.e. the human face, thus requiring features or descriptors having enough discriminating power necessary for a fine-grained accuracy.

In mathematics, spherical harmonics [49], [50] are a set of solutions to Laplace's equation in spherical coordinates, that form a set of basis functions of an orthogonal space:

$$
Y_{l}^{m}(\theta, \varphi)=K_{l}^{m} p_{l}^{m}(\cos \theta) e^{i m \varphi}
$$

where the symbol $p_{l}^{m}$ denotes the associated Legendre polynomials that are real-valued and defined over the range $[-1,1] . K_{l}^{m}$ is simply a scaling factor to normalize the functions:

$$
K_{l}^{m}=\sqrt{\frac{(2 l+1)}{4 \pi} \frac{(l-m) !}{(l+m) !}}
$$

3D face in spherical depth map is a spherical function $f(\theta, \phi)$ which can be expanded into a linear combination of all spherical harmonics scaled by their associated coefficients $h_{l} m$ :

$$
f(\theta, \varphi)=\sum_{l=0}^{\infty} \sum_{m=-l}^{l} h_{l m} Y_{l}^{m}(\theta, \varphi)
$$

Considering that 3D FR only requires real valued spherical harmonics, the equation of real Spherical Harmonics can be set forth as follows:

$$
y_{l}^{m}(\theta, \varphi)=\left\{\begin{array}{l}
\sqrt{2} K_{l}^{m} \cos (m \varphi) p_{l}{ }^{m}(\cos \theta), m>0 \\
\sqrt{2} K_{l}{ }^{m} \sin (-m \varphi) p_{l}{ }^{-m}(\cos \theta), m<0 \\
K_{l}^{0} p_{l}^{0}(\cos \theta), m=0
\end{array}\right.
$$

where $p_{l}^{m}$ and $K_{l}^{m}$ are the same as Equation (1). 
Finally, the associated coefficients $h_{l} m$, i.e. the spherical harmonic features (SHF), are obtained by the following equation:

$$
h_{l m}=\int_{0}^{2 \pi} \int_{0}^{\pi} f(\theta, \varphi) y_{l}{ }^{m}(\theta, \varphi) \sin \theta d \theta d \varphi
$$

where $\sin \theta$ is a regulatory factor for the integral in spherical coordinates. This equation is suitable for symbolic integration. The integral has to be evaluated numerically using Monte Carlo integration [51]. The numerical solution for the above integral problem can then be rewritten as:

$$
c_{l m}=\frac{1}{n} \sum_{j=1}^{n} f\left(x_{j}\right) y_{l}{ }^{m}\left(x_{j}\right) 4 \pi=\frac{4 \pi}{n} \sum_{j=1}^{n} f\left(x_{j}\right) y_{l}{ }^{m}\left(x_{j}\right)
$$

where $n$ is the number of sample points on $f(\theta, \phi)$, i.e. the input 3D face model. $4 \pi$ is a weight in the Monte Carlo estimator for every sample with equal probability.

In the end, a 3D face model is thus characterized by the energies at different frequencies of spherical harmonics. The energies at low frequencies capture the gross shape of the facial surface whereas those at high frequencies describe the fine facial surface details. As a result, these SHFs make it possible to capture both global and local facial shape information. This is in clear contrast to most 3D FR techniques known so far in the literature, which are either holistic, thus based on a global facial representation, or feature-based making use of local features extracted from a number of distinctive points.

\section{FeAture SELECTion}

As SHFs at different frequencies capture different geometric properties of a given facial surface, with the energies at low frequencies capturing the gross shape while those at high frequencies describe the fine details, and given the fact that 3D face scans can convey variations due to facial expression and partial occlusion, we proceed to learning the spherical harmonic features (SHF) which best discriminate the identities of 3D face scans through a feature selection method.

Feature selection, also known as variable selection or attribute selection, aims at selecting an optimal subset of discriminative features for building a robust classifier. The purpose of feature selection is three-fold: improving the prediction performance of the predictors, providing faster and more cost-effective predictors, and providing a better understanding of the underlying process 
that generated the data [6]. Based on the relations with respect to predictors, feature selection algorithms broadly fall into two categories: filter and embedded methods. The filter methods take into account the general properties of training data and evaluate the relevance of features without involving any predictor algorithm, whilst the embedded methods incorporate feature selection as part of the training process, and the feature relevance is evaluated analytically according to the associated predictor. In this work, three representative methods for feature selection, namely Relief-F [52], mRmR [53] (filter model) and Random Forests [54] (embedded model), are investigated. These three methods are different from each other as they achieve the selection of discriminating features through different properties from the training data. Our aim is to discover which kind of technique is most suitable for the selection of the most discriminating SHFs.

\section{A. Relief-F}

Relief-F is a type of supervised feature weighting algorithm which belongs to the category of filter methods. The key idea of Relief-F is to estimate the contribution of features according to their ability to distinguish between the nearest instances. The scheme of Relief-F is summarized as follows:

(0) Initialize $W(a)=0$ for each feature $a . m$ is a user-defined integer.

(1) Randomly select an instance $R_{i}$.

(2) Find $k$ nearest neighbors $H_{j}$ from the same class with $R_{i}$.

(3) Find $k$ nearest neighbors $M_{j}(C)$ from each class $C \neq \operatorname{class}\left(R_{i}\right)$.

(4) For each feature $a$, calculate the corresponding weight $W(a)$ through the following equation:

$$
\begin{aligned}
W(a) & =W(a)-\sum_{j=1}^{k} d\left(a, R_{i}, H_{j}\right) /(m \cdot k) \\
& +\sum_{C}\left[K \sum_{j=1}^{k} d\left(a, R_{i}, M_{j}(C)\right)\right] /(m \cdot k) \\
K & =\frac{P(C)}{1-P\left(\operatorname{class}\left(R_{i}\right)\right)}
\end{aligned}
$$

where $d\left(a, I_{1}, I_{2}\right)$ calculates the difference between two instances $I_{1}$ and $I_{2}$ with respect to feature a. $P(C)$ is the prior probability of class $C .1-P\left(\operatorname{class}\left(R_{i}\right)\right)$ denotes the sum of probabilities for class $C \neq \operatorname{class}\left(R_{i}\right)$. 
(5) Repeat the above process from (1) for $m$ times. Finally return the weight vector $W$ of all features.

\section{B. minimal-Redundancy-maximal-Relevance}

$\mathrm{mRmR}$ selects a subset of best features according to the criterion of minimal-redundancymaximal-relevance based on mutual information. Given two variables $x$ and $y$, their mutual information can be defined as:

$$
I(x, y)=\iint p(x, y) \log \frac{p(x, y)}{p(x) p(y)} d x d y
$$

where $p(\cdot)$ is the probability density function. The criterion of maximal-relevance is to select features $S$ satisfying the following equation:

$$
\max D(S, c), D(S, c)=\frac{1}{|S|} \sum_{x_{i} \in S} I\left(x_{i}, c\right)
$$

where $c$ is a class label. However, it is well known that the combination of individually good features does not necessarily lead to good classification performance because the features selected according to maximal-relevance could have rich redundancy. To remedy this drawback, minimalredundancy criterion is added to reduce the redundancy between features:

$$
\min R(S), R(S)=\frac{1}{|S|^{2}} \sum_{x_{i}, x_{j} \in S} I\left(x_{i}, x_{j}\right)
$$

The combination of the above two criteria is $\max (D-R)$. In practice, incremental search method is normally used to select the near optimal subset of features. Given the selected subset $S_{m-1}$, the $m$ th feature can be selected from the set $\left\{X-S_{m-1}\right\}$ by the following equation:

$$
\max \left[I\left(x_{j}, c\right)-\frac{1}{m} \sum_{x_{i} \in S_{m-1}} I\left(x_{j}, x_{i}\right)\right], x_{j} \in X-S_{m-1}
$$

\section{Random Forests}

Breiman [54] gives the definition of Random Forests as a classifier consisting of a collection of decision trees $\left(h\left(x, \Theta_{k}\right), k=1,2, \cdots\right)$, where $\Theta_{k}$ are independent identically distributed random vectors. Each decision tree casts a unit vote for the final output with respect to input $\mathrm{x}$. The core task of Random Forests is the construction of each decision tree. Further details can be found in $[54]$. 


\section{EXPERIMENTAL RESUlts AND ANALysis}

The effectiveness of the proposed method is evaluated in this section. First, we shortly describe the three datasets on which the experiments were carried out. We then evaluate the discriminating power of the proposed spherical harmonic features (SHF). This is followed by experiments highlighting the usefulness of feature selection methods. Finally, 3D FR experiments using selected SHF are performed and the results compared with the state of the art.

\section{A. Face Databases}

Our experiments are conducted on three popular face datasets, namely SHREC2007 [36], FRGC2.0 [1] and Bosphorus [55]. Table I gives a comparative overview of these three datasets.

TABLE I

A COMPARATIVE OVERVIEW OF THE THREE PUBLIC 3D FACE DATASETS.

\begin{tabular}{|l|c|c|c|}
\hline Databases & SHREC2007 & FRGC2.0 & Bosphorus \\
\hline Subject & 64 & 466 & 105 \\
\hline Total & 640 & 4007 & 4666 \\
\hline Expression & neutral & $\begin{array}{c}\text { smile } \\
\text { surprise } \\
\text { angry }\end{array}$ & 35 expressions \\
\hline Pose & NA & NA & 13 yaw, pitch, \\
cross rotations \\
\hline Occlusion & NA & NA & 4 occlusions \\
\hline
\end{tabular}

Because the computation of Spherical Harmonic Feature (SHF) at different frequencies through Equation (5) and (6) requires the global description of a 3D shape, 3D face scans having only the half face in Bosphorus are excluded from our experiments. Therefore, a total of 3954 faces from 105 subjects in Bosphorus are used in the experiments.

\section{B. Spherical Harmonic Features}

As described in section III, there exists an infinity of spherical harmonics at different frequencies. Therefore, the computation of all SHFs is infeasible and one needs to find out a tradeoff between the descriptive power of SHFs and the associated computational cost. In this work, we 
have chosen $l=(1 \sim 25)$, which yields $(25+1)^{2}=676$ coefficients, along with the Simple euclidian distance to compute the similarity of two SHFs.

In order to test the discriminating power of SHF which is now approximated by a 676 dimensional feature vector, we carried out face verification experiment on the three aforementioned datasets, that is to say, the query set and the target set are all the faces in each dataset. Fig. 8 gives the ROC curves of the proposed SHF. The verification rate on the three datasets at FAR $=0.001$ is $92 \%, 90 \%$ and $81.4 \%$, for SHREC2007, FRGC2.0 and Bosphorus respectively.

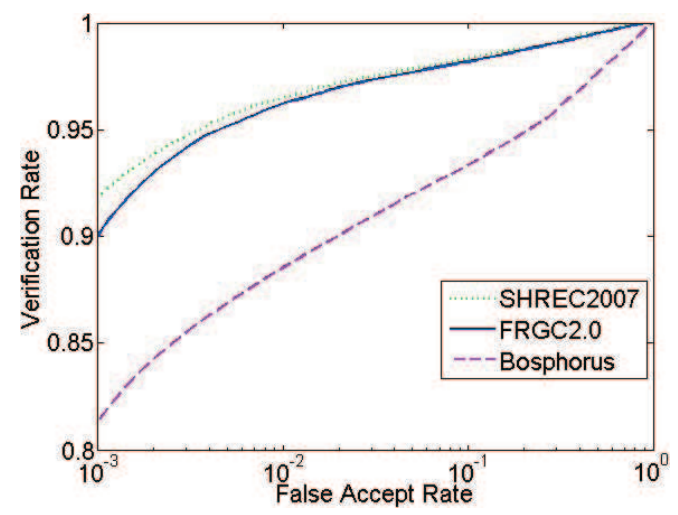

Fig. 8. ROC curves of SHF on three datasets.

We compared SHF with some state-of-the-art methods on the FRGC2.0 dataset. All experiments were performed under the same conditions, i.e. under "All versus All" partition. Table II shows the comparison of verification rate at FAR $=0.001$. Our approach achieves the highest verification rate when using a single feature.

\section{Learning the Discriminating Spherical Harmonic Features}

The next step is to test the usefulness of feature selection methods. Given that learning is required for these feature section methods, it is important to check their skill of generalization on unseen subjects [33]. To this end, we divided the subjects used for training and testing into two disjoint sets in the experiments as shown in Table III. Given that the three datasets are organized by subject and each subject has a similar distribution, our divisions were based on subject. For example, the 64 subjects with 640 face scans of SHREC2007 were split into a 
TABLE II

THE COMPARISON OF VERIFICATION RATE AT FAR $=0.001$ USING THE FRGC2.0 DATABASE.

\begin{tabular}{|l|c|c|}
\hline & Single feature-mode & Fusion \\
\hline Maurer et al. [56] & $77.6 \%$ & $86.9 \%$ \\
\hline Cook et al. [57] & - & $92.31 \%$ \\
\hline Mian et al. [58] & $72.55 \%, 74.31 \%$ & $86.6 \%$ \\
\hline Faltemier et al. [59] & $<84.8 \%$ & $93.2 \%$ \\
\hline Queirolo et al. [37] & - & $96.5 \%$ \\
\hline Wang et al. [21] & - & $98.13 \%$ \\
\hline Berretti et al. [34] & $81.2 \%$ & - \\
\hline Our method & $90 \%$ & - \\
\hline
\end{tabular}

training and testing set of equal size, each having 320 face scans of 32 subjects. The other two databases were divided in the same way.

TABLE III

DISJOINT SETS FOR TRAINING AND TESTING. THE NUMBERS IN THE TABLE ARE LISTED IN TERMS OF FACE SCANS/SUBJECTS.

\begin{tabular}{|l|c|c|c|}
\hline Databases & SHREC2007 & FRGC2.0 & Bosphorus \\
\hline Training set & $320 / 32$ & $1973 / 170$ & $1977 / 58$ \\
\hline Test set & $320 / 32$ & $2034 / 296$ & $1977 / 47$ \\
\hline
\end{tabular}

Since Relief-F is a feature weighting algorithm [52], a feature list ranked by weight, representing the discriminating ability of the corresponding feature, is returned by Relief-F. To evaluate the effectiveness of Relief-F, KNN classifier was conducted on the selected feature set by incrementally increasing the number of features used on the test set until the full list of features was reached. For each dataset, 10-fold cross validation was used to guarantee the reliability of the results. Fig.9 shows the accuracy curves on the three datasets. For the purpose of comparison in order to highlight the generalization skill of the chosen features, accuracy on the training sets was also calculated in the same manner.

Minimum-Redundancy-Maximum-Relevance $(\mathrm{mRmR})$ selects a subset of features that have higher correlation to the prediction performance [53]. In this study, 300 variables ranked by the correlation were selected from the 676 Spherical Harmonic Features. The effectiveness of these 

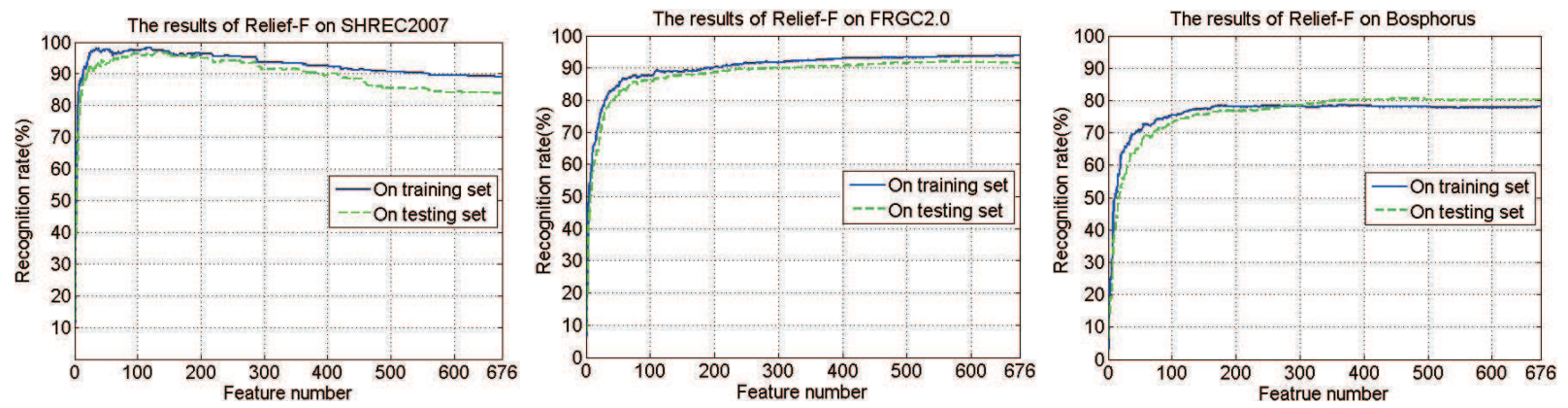

Fig. 9. Performance evaluation of Relief-F on the three datasets by 10 -fold cross validation using the KNN classifier.

features was then evaluated in the same way as Relief-F, as shown in Fig.10.
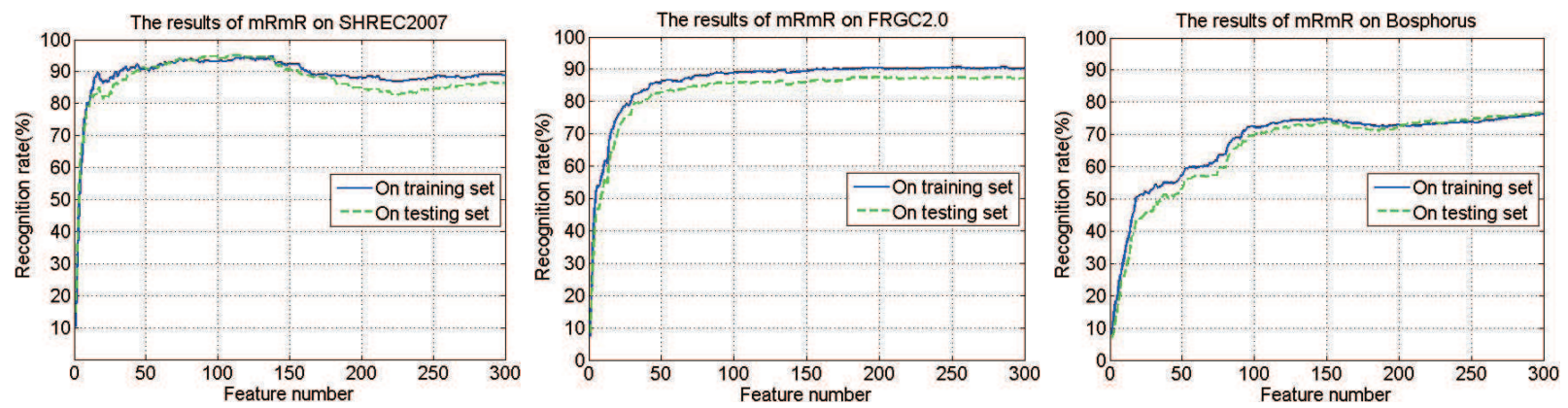

Fig. 10. Performance evaluation of $\mathrm{mRmR}$ on the three datasets by 10 -fold cross validation using the KNN classifier.

By observing the curves in figure 9 and 10, we can draw the following conclusions:

- By comparing the results between the training set and testing set, it can be seen that the features selected by Relief-F and $\mathrm{mRmR}$ have a very good generalization skill on unseen subjects.

- The features selected by Relief-F and mRmR perform the best on SHREC2007, then on FRGC v2.0 and lastly on Bosphorus. This accords with the increasing difficulties of these three datasets in terms of face data, face expression variations, pose changes and even occlusion.

- Relief-F performs consistently better than $\mathrm{mRmR}$ on the three datasets.

As we can see from Fig. 9 and 10, the recognition accuracy increases quite quickly and attains a point of inflection when the number of features selected by both Relief-F and $\mathrm{mRmR}$ is augmented. This recognition accuracy then remains stable with very slight improvement in the 
case of FRGC v2.0 and Bosphorus or even decreases in the case of SHREC2007 when the number of selected features is further increased. The selection of relevant features in our case thus requires a tradeoff between computational complexity and recognition accuracy. While the selection of more features may lead to better recognition accuracy, it also leads to a higher computational complexity of the predictor. Many solutions to this question exist, e.g., second derivative or Receiver Operating Characteristic analysis, which automatically determine the number of selected features to be used based on the requirements of the application in hand. In this work, based on the performance of the features selected by Relief-F and $\mathrm{mRmR}$ on the testing set as shown on Fig. 9 and 10, we simply manually selected the subset of features being used in the three datasets. Table IV summarizes the number of selected features in each subset, per dataset and per feature selection method.

TABLE IV

THE NUMBER OF SELECTED FEATURES IN EACH FEATURE SUBSET.

\begin{tabular}{|l|c|c|c|}
\hline Databases & SHREC2007 & FRGC2.0 & Bosphorus \\
\hline Relief-F & 148 & 188 & 171 \\
\hline mRmR & 135 & 168 & 153 \\
\hline
\end{tabular}

These feature subsets were then used in the task of face recognition.

\section{Experiments on $3 D F R$}

To validate the improvements of feature selection on 3D FR, both verification and identification experiments were carried out. There are a series of well-known protocols in FRGC2.0 for face recognition experiments. Unfortunately they are only suitable for FRGC2.0 data. As a result, we designed some more general experiment protocols in order to enable the comparison of the performance of the proposed methods on the three datasets. These experiment protocols are first explained and are then followed by the experimental figures.

For face verification, the query set and the target set were all faces from the three testing sets. Therefore, the size of the similarity matrixes on the three datasets is $320 \times 320,2034 \times 2034$ and $1977 \times 1977$, respectively. Fig. 11 gives the ROC curves of the feature subsets by Relief-F and $\mathrm{mRmR}$ in comparison with all features on the three test sets. As we can see from these curves, Relief-F once again outperforms $\mathrm{mRmR}$ and the use of all features. 


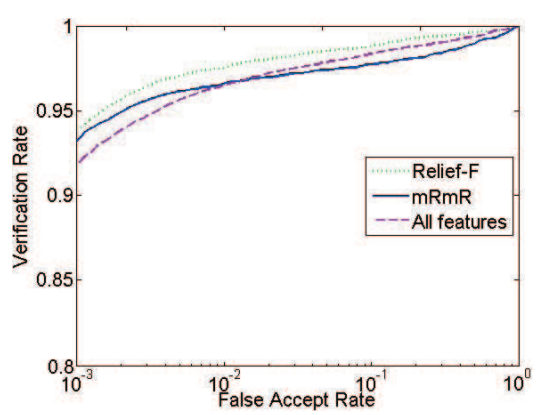

(a) SHREC2007

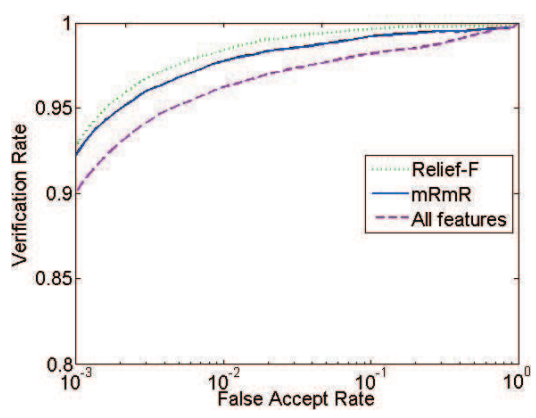

(b) FRGC2.0

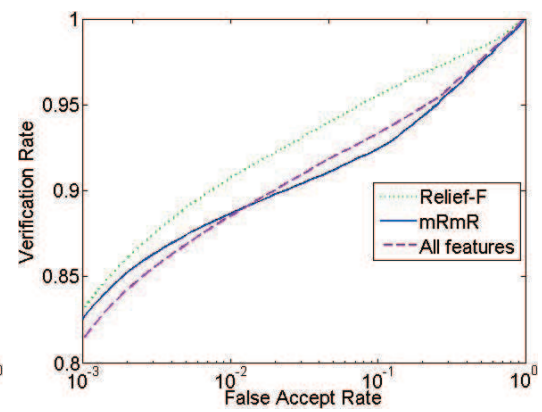

(c) Bosphorus

Fig. 11. The comparison of ROC curves between Relief-F, mRmR and All Features.

For face identification, 10-fold cross validation using KNN classifier was employed to calculate the identification rate on the three datasets. Furthermore, this accuracy was compared with 10fold cross validation using SVM classifier. Table V shows the comparison of recognition rates. The results of Random Forests are listed in the last line of the table.

TABLE V

THE COMPARISON OF IDENTIFICATION RATES. THE IDENTIFICATION RATES USING ALL SHF ARE ALSO CALCULATED AS THE BASELINE.

\begin{tabular}{|l|c|c|c|}
\hline & SHREC2007 & FRGC2.0 & Bosphorus \\
\hline KNN(all) & $89.75 \%$ & $88.24 \%$ & $75.87 \%$ \\
\hline SVM(all) & $97.24 \%$ & $96.85 \%$ & $94.42 \%$ \\
\hline Relief-F+KNN & $96.53 \%$ & $88.25 \%$ & $76.61 \%$ \\
\hline Relief-F+SVM & $97.86 \%$ & $96.94 \%$ & $95.63 \%$ \\
\hline mRmR+KNN & $94.99 \%$ & $86.27 \%$ & $73.92 \%$ \\
\hline mRmR+SVM & $96.47 \%$ & $89.52 \%$ & $87.79 \%$ \\
\hline RF & $96.83 \%$ & $92.97 \%$ & $89.22 \%$ \\
\hline
\end{tabular}

As we can see from Table $\mathrm{V}$, the best results, respectively $97.86 \%, 96.94 \%$ and $95.63 \%$ on the three datasets, were achieved by Relief-F+SVM. Remember that SHREC2007, FRGC v2.0 and Bosphorus, as summarized in table I, are three datasets of increasing difficulties in terms of the number of subjects, facial expression, pose and occlusion. Under the combined effect of the discriminating power as provided by SHF and the feature selection method, the recognition rate by Relief+SVM only decreases slightly on FRGC v2.0 with facial expression variations and 
Bosphorus with pose changes, facial expression variations and occlusions. These experimental results demonstrate the effectiveness of the proposed method. In addition, the computation cost is significantly reduced by using feature selection methods. The average time for the computation of $l=(1 . .25)$ band SHF (676 features) of a face scan in FRGC2.0 decreases from 2.72 seconds to 0.8 seconds, thereby cutting the computational cost by a factor of 3 . The experiments were implemented using Matlab on a PC with Intel(R) Core(TM)2 Quad CPU $2.40 \mathrm{GHz}$ CPU and 4G RAM. Therefore, there is still room to further acceleration of the computation process.

\section{CONCLUSiONS}

In this paper a novel competitive method for 3D FR has been proposed, which is based on Spherical Harmonic Features (SHF) reliably computed on a canonical 3D face representation, namely Spherical Depth Map (SDM). Feature selection methods are further used to improve the predictive performance and provide faster and more cost-effective predictors. The experimental results on three public 3D face datasets, namely SHREC2007, FRGC v2.0 and Bosphorus, highlight the discriminating power of the proposed Spherical Harmonic Features. Although there is a slight decrease in performance when expression and occlusion are taken into account, proper feature selection method can compensate for this defect. Finding or designing better feature selection method for SHF will be our future objective.

\section{ACKNOWLEDGMENT}

This work is in part funded by the National Basic Research Program of China (No. 2010CB327902), the National Natural Science Foundation of China (No. 60873158, No. 61005016, No. 61061130560), the Sino-French project 3D Face Analyzer supported by the French research agency, Agence Nationale de Recherche (ANR), through the grant ANR 2010 INTB 0301 01, and the 3D Face Interpreter project supported by the LIA 2MCSI lab between the group of Ecoles Centrale and Beihang University.

\section{REFERENCES}

[1] P. J. Phillips, P. J. Flynn, T. Scruggs, K. W. Bowyer, J. Chang, K. Hoffman, J. Marques, J. Min, and W.Worek, "Overview of the face recognition grand challenge," in IEEE CVPR, 2005, pp. 947-954.

[2] N. Pears, T. Heseltine, and M. Romero, "From 3D point clouds to pose-normalised depth maps," IJCV, vol. 89, pp. 152-176, 2010. 
[3] L. Akarun, B. Gokberk, and A. Salah, “3D face recognition for biometric applications,” in Proc. European Signal Processing Conference, 2005.

[4] K. Bowyer, K. Chang, and P. Flynn, "A survey of approaches and challenges in 3D and multi-modal 3D + 2D face recognition," Computer Vision and Image Understanding, vol. 101, pp. 1-15, 2006.

[5] B. Gokberk, A. A. Salah, N. Alyuz, and L. Akarun, "3D face recognition: Technology and applications," in Handbook of Remote Biometrics. Springer, London, 2009, pp. 217-246.

[6] I. Guyon and A. Elisseeff, "An introduction to variable and feature selection," Journal of Machine Learning Research, vol. 3, pp. 1157-1182, 2003.

[7] A. L. Blum and P. Langley, "Selection of relevant features and examples in machine learning," Artificial Intelligence, vol. 97, pp. 245-271, 1997.

[8] R. Kohavi and G. H. John, "Wrappers for feature subset selection,” Artificial Intelligence, vol. 97, pp. $273-324,1997$.

[9] H. Liu and H. Motoda, "Computational methods of feature selection." Chapman and Hall/CRC, 2007.

[10] B. K. P. Horn, “Extended gaussian images," Proceedings of the IEEE, vol. 72, no. 2, pp. 1671-1686, 1984.

[11] C. Dorai and A. K. Jain, "Cosmos-a representation scheme for 3D free-form objects," IEEE Trans. PAMI, vol. 19, no. 10, pp. 1115-1130, 1997.

[12] A. E. Johnson, "Spin-images: A representation for 3-D surface matching." Ph. D. Thesis, Carnegie Mellon University, 1997.

[13] M. Ankerst, G. Kastenmuller, H. Kriegel, and T. Seidl, "3D shape histograms for similarity search and classification in spatial databases," in SSD'99, 1999, pp. 207-226.

[14] R. Osada, T. A. Funkhouser, B. Chazelle, and D. P. Dobkin, "Shape distributions," ACM Trans. Graph, vol. 21, no. 4, pp. 807-832, 2002.

[15] M. M. Kazhdan, T. A. Funkhouser, and S. Rusinkiewicz, "Rotation invariant spherical harmonic representation of 3D shape descriptors.” in Symposium on Geometry Processing'03, 2003, pp. 156-165.

[16] H. Wendland, "Scattered data approximation.” Cambridge University Press, New York, 2005.

[17] A. M. Bronstein, M. M. Bronstein, and R. Kimmel, "Expression-invariant representations of faces," IEEE Trans. on Image Processing, vol. 16, no. 1, pp. 188-197, 2007.

[18] I. A. Kakadiaris, G. Passalis, G. Toderici, M. N. Murtuza, Y. Lu, N. Karampatziakis, and T. Theoharis, "Three-dimensional face recognition in the presence of facial expressions: An annotated deformable model approach," IEEE Trans. Pattern Anal. Mach. Intell., vol. 29, no. 4, pp. 640-649, 2007.

[19] C. Samir, A. Srivastava, M. Daoudi, and E. Klassen, "An intrinsic framework for analysis of facial surfaces," International Journal of Computer Vision, vol. 82, no. 1, pp. 80-95, 2009.

[20] M. H. Mahoor and M. Abdel-Mottaleb, "Face recognition based on 3D ridge images obtained from range data," Pattern Recognition, vol. 42, no. 3, pp. 445-451, 2009.

[21] Y. Wang, J. Liu, and X. Tang, "Robust 3D face recognition by local shape difference boosting," IEEE Trans. PAMI, vol. 32, no. 10, pp. $1858-1870,2010$.

[22] D. Huang, M. Ardabilian, Y. Wang, and L. Chen, "A novel geometric facial representation based on multi-scale extended local binary patterns," in 9th IEEE Conference on Automatic Face and Gesture Recognition, 2011.

[23] B. Gokberk, M. O. Irfanoglu, and L. Akarun, "3D shape-based face representation and feature extraction for face recognition," Image and Vision Computing, vol. 24, no. 8, pp. 857-869, 2006.

[24] T. S. Jebara, “3D pose estimation and normalization for face recognition.” B. E. Thesis, McGill University, 1996. 
[25] G. G. Gordon, "Face recognition based on depth maps and surface curvature," in SPIE Geometric methods in Computer Vision, 1991, pp. 234-247.

[26] D. Colbry, G. Stockman, and A. K. Jain, "Detection of anchor points for 3D face verification," in IEEE CVPR, 2005.

[27] C. Conde and A. Serrano, "3D facial normalization with spin images and influence of range data calculation over face verification," in IEEE CVPR, 2005.

[28] X. Tang, J. Chen, and Y. Moon, "Towards more accurate 3D face registration under the guidance of prior anatomical knowledge on human faces," in IEEE International Conference on Automatic Face and Gesture Recognition, 2008, pp. 1-6.

[29] F. B. Haar and R. C. Veltkamp, "A 3D face matching framework," in IEEE International Conference on Shape Modeling and Applications, 2008, pp. 103-110.

[30] F. Al-Osaimi, M. Bennamoun, and A. Mian, "An expression deformation approach to non-rigid 3D face recognition," International Journal of Computer Vision, vol. 81, no. 3, pp. 302-316, 2009.

[31] C. Boehnen, T. Peters, and P. J. Flynn, “3D signatures for fast 3D face recognition," in Third International Conference on Advances in Biometrics, 2009, pp. 12-21.

[32] C. Xu, S. Li, T. Tan, and L. Quan, "Automatic 3D face recognition from depth and intensity gabor features," Pattern Recognition, vol. 42, no. 9, pp. 1895-1905, 2009.

[33] R. S. Llonch, E. Kokiopoulou, I.Tosic, and P.Frossard, “3D face recognition with sparse spherical representations," Pattern Recognition, vol. 43, no. 3, pp. 824-834, 2010.

[34] S. Berretti, A. D. Bimbo, and P. Pala, "3D face recognition using isogeodesic stripes," IEEE Trans. PAMI, vol. 32, no. 12, pp. 2162-2177, 2010.

[35] D. Huang, W. B. Soltana, M. Ardabilian, Y.Wang, and L.Chen, "Textured 3D face recognition using biological vision-based facial representation and optimized weighted sum function," in IEEE CVPR 2011 Workshop on Biometrics, 2011.

[36] "Shape retrieval contest of 3D face models." http://give-lab.cs.uu.nl/SHREC/shrec2007/, 2007.

[37] C. C. Queirolo, L. Silva, O. Bellon, and M. P. Segundo, "3D face recognition using simulated annealing and the surface interpenetration measure," IEEE Trans. PAMI, vol. 32, no. 2, pp. 206-219, 2010.

[38] V. Vijayan, K. W. Bowyer, P. J. Flynn, D. Huang, L. Chen, M. Hansen, O. Ocegueda, S. K. Shah, and I. A. Kakadiaris, “Twins 3D face recognition challenge," in International Joint Conference on Biometrics, 2011.

[39] A. S. Mian, M. Bennamoun, and R. A. Owens, "Keypoint detection and local feature matching for textured 3d face recognition," International Journal of Computer Vision, vol. 79, no. 1, pp. 1-12, 2008.

[40] R. C. Veltkamp, S. van Jole, H. Drira, B. B. Amor, M. Daoudi, H. Li, L. Chen, P. Claes, D. Smeets, J. Hermans, D. Vandermeulen, and P. Suetens, "Shrec '11 track: 3d face models retrieval," in 3DOR, 2011, pp. 89-95.

[41] T. A. Funkhouser, P. Min, M. M. Kazhdan, J. Chen, J. A. Halderman, D. P. Dobkin, and D. P. Jacobs, "Search engine for 3D models," ACM Transactions on Graphics, vol. 22, pp. 83-105, 2003.

[42] P. Liu, Y. Wang, Z. Zhang, and Y. Wang, "Automatic and robust 3D face registration using multiresolution spherical depth map," in International Conference on Image Processing. IEEE, 2010, pp. 4389-4392.

[43] Y. Saeys, I. Inza, and P. Larranaga, "A review of feature selection techniques in bioinformatics," Bioinformatics, vol. 23, no. 19 , pp. 2507-2517, 2007.

[44] P. Liu, Y. Wang, and Z. Zhang, "Representing 3D face from point cloud to face-aligned spherical depth map," International Journal of Pattern Recognition and Articial Intelligence, vol. 26, no. 1, p. Online, 2012. 
[45] P. J. Besl and N. D. Mckay, "A method for registration of 3D shapes," IEEE Trans. PAMI, vol. 14, no. 2, pp. 239-256, 1992.

[46] P. Szeptycki, M. Ardabilian, and L. Chen, "A coarse-to-fine curvature analysis-based rotation invariant 3d face landmarking," in Proceedings of the 3rd IEEE international conference on Biometrics: Theory, applications and systems, ser. BTAS'09. Piscataway, NJ, USA: IEEE Press, 2009, pp. 32-37. [Online]. Available: http://dl.acm.org/citation.cfm?id=1736406.1736411

[47] X. Zhao, E. Dellandréa, L. Chen, and I. A. Kakadiaris, "Accurate landmarking of three-dimensional facial data in the presence of facial expressions and occlusions using a three-dimensional statistical facial feature model," IEEE Transactions on Systems, Man, and Cybernetics, Part B, vol. 41, no. 5, pp. 1417-1428, 2011.

[48] Y. Liu, K. L. Schmidt, J. F. Cohn, and S. Mitrac, "Facial asymmetry quantification for expression invariant human identification," Computer Vision and Image Understanding, vol. 91, no. 1-2, pp. 138-159, 2003.

[49] V. Schonefeld, "Spherical Harmonics.” http://limbicsoft.com/volker/prosem_paper.pdf, 2005.

[50] W. Freeden, T. Gervens, and M. Schreiner, "Constructive approximation on the sphere with applications to geomathematics." CLARENDON PRESS, OXFORD, 1998.

[51] R. E. Caflisch, "Monte carlo and quasi-monte carlo methods," Acta Numerica, vol. 7, pp. 1-49, 1998.

[52] M. Robnik-Sikonja and I. Kononenko, "Theoretical and empirical analysis of ReliefF and RReliefF," Machine Learning Journal, vol. 53, pp. 23-69, 2003.

[53] H. Peng, F. Long, and C. Ding, "Feature selection based on mutual information: Criteria of Max-Dependency, MaxRelevance, and Min-Redundancy," IEEE Transactions on Pattern Analysis and Machine Intelligence, vol. 27, no. 8, pp. 1226-1238, June 2005.

[54] L. Breiman, "Random forests,” Machine Learning, vol. 45, pp. 5-32, 2001.

[55] A. Savran, N. Alyuz, H. Dibeklioglu, O. Celiktutan, B. Gokberk, B. Sankur, and L. Akarun, "Bosphorus database for 3D face analysis," in The First COST 2101 Workshop on Biometrics and Identity Management. Roskilde University, 2008.

[56] T. Maurer, D. Guigonis, I. Maslov, B. Pesenti, A. Tsaregorodtsev, D. West, and G. Medioni, "Performance of Geometrix ActiveID $^{T M}$ 3D face recognition engine on the FRGC data," in IEEE CVPR, 2005.

[57] J. Cook, V. Chandran, and C. Fookes, “3D face recognition using log-gabor templates," in BMVC'06, 2006, pp. 769-778.

[58] A. S. Mian, M. Bennamoun, and R. Owens, "An efficient multimodal 2D-3D hybrid approach to automatic face recognition," IEEE Trans. PAMI, vol. 29, no. 11, pp. 1927-1943, 2007.

[59] T. C. Faltemier, K. W. Bowyer, and P. J. Flynn, “A region ensemble for 3-D face recognition,” IEEE Trans. on Information Forensics and Security, vol. 3, no. 1, pp. 62-73, 2008. 